\title{
Género y sufijos clasificatorios de discretización en ocaina (witoto)
}

\author{
Gender and classificatory suffixes of discretization \\ in Ocaina (Witoto)
}

Doris Fagua Rincón

\begin{abstract}
Resumen
En ocaina coexisten marcas de género y sufijos clasificatorios de discretización ( $c f$. clasificadores), rasgo areal y tipológicamente poco documentado. El género clasifica el léxico nominal en animado $v s$ inanimado y no se manifiesta directamente sobre el nombre mismo sino como una marca de concordancia: formas pronominales. La segunda categoría se manifiesta en la morfología de la subclase de nombres genéricos y densos ( $c f$. nombres no contables). No se trata sin embargo de un dispositivo clasificatorio de los nombres sino que más bien clasifica la manera como los nombres así especificados pueden ser discretizados: por ello se habla de sufijos "clasificatorios de discretización". Este paradigma corresponde a una de las formas de marcar la categoría de número singular. Distinguimos entre "género" o "género gramatical", categoría que constituye un dispositivo nominal clasificatorio; y "género semántico": masculino vs femenino, correspondientes a dos marcas que hacen parte del paradigma de sufijos discretizadores (categoría de número).

Palabras clave: Género. Sufijos clasificatorios. Ocaina. Witoto. Amazonia.
\end{abstract}

\begin{abstract}
Ocaina has both gender markers and classificatory discretization suffixes ( $c f$. classifiers), areal and typological feature under-documented. Gender classifies the nominal lexicon into animate $v s$ inanimate and reflects beyond the noun itself through agreement: pronominal forms. The second category appears directly on the morphology of the subclasses generic and mass nouns ( $c f$. uncountable nouns). It is not a classificatory noun device but the way in which nouns specified with these suffixes became discret: for this reason they are told "clasificatory discretization" suffixes. This paradigm is one of the means marking the singular number category. We distinguish the "gender" or "grammatical gender" category, a classificatory device, from "semantic gender": masculin $v s$ femenin, marks that belong to the paradigm of discretizer suffixes (number category).

Keywords: Gender. Classificatory suffixes. Ocaina. Witotoan. Amazon.
\end{abstract}

Los ocaina hacen parte del área o complejo cultural y lingüístico de la Amazonia noroccidental conocido con las auto-denominaciones de Gente de Centro e Hijos del tabaco, la coca y la yuca dulce, al cual pertenecen los grupos etnolingüísticos: bora, muinane (bora), huitoto, nonuya, ocaina (witoto), resígaro (arawak) y andoque (lengua aislada). Estas poblaciones se encuentran

\footnotetext{
${ }^{1}$ Buena parte del corpus que dio lugar a los análisis aquí expuestos provienen de Seifart, F., Fagua, D., Gasché, J. et al. (2009). Expresamos nuestra gratitud a Rogelio Andrade Nuñez, hablante de ocaina, por su asesoría paciente y entretenida.
} 
ubicadas principalmente en el interfluvio Caquetá-Putumayo y en la cuenca del río Ampiyacu, afluente del Amazonas, en Colombia y Perú, respectivamente. Siguiendo la clasificación de la $\mathrm{UNESCO}^{2}$, el ocaina es una lengua seriamente amenazada de extinción. Efectivamente, solo queda una cincuentena de hablantes fluidos de la generación de los abuelos y aproximadamente, otros cincuenta adultos entre los 35 y 45 años que poseen algún tipo de competencia para la comprensión y pasiva para la expresión. Por tal razón, esta lengua ya no es transmitida a los niños y ha sido desplazada por el castellano, que es hoy la lengua primera de las jóvenes generaciones. Uno de los últimos bastiones de esta lengua es el ámbito ritual y ceremonial.

En este artículo presentamos la existencia en una misma lengua de marcas de género y de sufijos clasificatorios de discretización ( $c f$. clasificadores), lo que constituiría un aspecto del ocaina areal y tipológicamente digno de interés, por haber sido poco documentado. Se trataría en efecto de un sistema mixto, siguiendo la propuesta de una tipología de los sistemas de clasificación nominal de Colette Grinevald (2004:261, 2002:260): un continuum de gramaticalización que iría de un polo lexical (términos de clase y de medida), pasando por los clasificadores, hasta un polo morfosintáctico (clases nominales y género). Queremos desde ahora aclarar que esta característica del ocaina, más que un rasgo original de la lengua, constituiría un análisis alternativo de los fenómenos ligados a la clasificación nominal, que podría extenderse a otras lenguas del área de la Amazonia noroccidental (Aikhenvald, 2011:28-29; Petersen, 2007: 389-409; Seifart \& Payne, 2007:381-387; Seifart, 2005:12).

Consideramos pertinente comenzar la presentación aclarando tanto la definición de género que adoptamos aquí como la distinción entre "género gramatical" y "género semántico". Siguiendo a Corbett (2007:241-243), el género o si se quiere "género gramatical" es una categoría a la cual todo nombre de una lengua es obligatoriamente atribuido y que se manifiesta en la concordancia. Esta categoría no implica necesariamente la presencia de marcas en el nombre. La concordancia es pues la base para la identificación de la categoría de género. La categoría género puede presentar dos o tres valores: masculino vs femenino; animado vs inanimado; masculino vs femenino vs neutro; también puede tratarse de un inventario de valores más amplio, como en el caso de la lenguas bantú, en las cuales el género correspondería a lo que en esa tradición descriptiva se denomina clases nominales.

El "género gramatical" -en adelante género a secas-, como vimos, es una categoría obligatoria manifiesta en la concordancia. Mientras que el "género semántico" es una distinción motivada entre sexos ( $c f$. hembra vs macho), que

${ }^{2}$ Atlas interactivo de la Unesco: http://www.unesco.org/culture/languages-atlas. 
no corresponde a una categoría nominal obligatoria. En ocaina, por ejemplo, la categoría de género según el rasgo de "animacidad" clasifica todo nombre de esta lengua ya sea como animado o bien como inanimado. En tanto que el género semántico corresponde a la dupla: -m̦a $\sim-m a$ 'masculino' y -ko -tfo 'femenino', marcas del inventario que conforma el paradigma de sufijos clasificatorios de discretización. Esta pareja de sufijos determina en particular, aunque no necesaria ni obligatoriamente, a humanos y animados (ver infra ejemplos: 2, 9; y cuadros 1 y 4).

\section{La clasificación nominal}

El tema general de la clasificación nominal remite en ocaina por una parte, a dos categorías gramaticales: el género (animado vs inanimado) y el número singular, que tiene como una de sus manifestaciones las marcas clasificatorias (cf. clasificadores), denominados aquí sufijos clasificatorios de discretización. Por otra parte, esas dos categorías están estrechamente relacionadas con las clases de nombres (ver cuadro 1). Así el género impone una división bipartita del léxico nominal: nombres animados vs inanimados. En función de la estrategia a la cual se recurre para indicar el número singular, los nombres se dividen pues en individuales, es decir nombres que remiten directamente a una unidad; y en genéricos y densos: aquellos a los que solo les es posible referir a unidades o elementos contables recurriendo a una operación de discretización, consistente en la sufijación de una marca clasificatoria (ver sección 2, infra).

Cuadro 1. Clasificación de los nombres en ocaina según las categorías de género y número singular

\begin{tabular}{l|l|l}
\hline CATEGORÍAS NOMINALES & CLASES DE NOMBRES & MARCAS \\
\hline Género & animados $v$ s inanimados & formas pronominales \\
\hline \multirow{5}{*}{ Número singular } & $\begin{array}{l}\text { individuales (cf. contables) } \\
v s\end{array}$ & $-\varnothing$ \\
\cline { 2 - 3 } & $\begin{array}{l}\text { genéricos y densos } \\
(c f . \text { no contables) }\end{array}$ & $\begin{array}{l}\text { sufijos clasificatorios } \\
\text { de discretización }\end{array}$ \\
\hline
\end{tabular}

\section{Nombres animados $v s$ inanimados: categoría de género}

Como mencionábamos arriba, todo nombre de la lengua es obligatoriamente atribuido a uno de los valores de la oposición binaria según el rasgo: +/-animado; y su manifestación de la es la concordancia, por ello hablamos de la categoría de género. En ocaina, esta categoría no necesariamente aparece marcada en la morfología nominal; se manifiesta, en cambio, obligatoriamente en correlatos morfosintácticos externos al núcleo nominal que controla la concordancia. Tales correlatos son formas pronominales que retoman los nombres en cuestión: 
como pronombres personales o bien, como índices pronominales prefijados a verbos, nombres y posposiciones. Puesto que el género solo se manifiesta en esas formas, esta categoría podría denominarse más precisamente "género pronominal"' (Corbett, 2007:242).

La oposición según la categoría de género solamente se marca en la tercera persona, singular y plural, ya que las demás personas por referir, en principio, a humanos son siempre animadas. Las variantes alomórficas de la tercera persona, inanimada son: $t^{j} a-, t^{j} \tilde{a} \tilde{a} \sim t^{j} \tilde{a} \tilde{a} h \tilde{u}$, se trata de una única forma para singular y plural.

Los siguientes ejemplos ${ }^{3}$ ilustran la manifestación de la concordancia a través de formas pronominales que retoman nombres mencionados anteriormente en el discurso o inferibles a partir del contexto de enunciación. En (1) aparece un pronombre objeto inanimado y dos índices pronominales prefijados a un verbo trivalente, que están desempeñando respectivamente las funciones objeto (indirecto) y sujeto.
(1) t่ẵăhũ $\tilde{\mathrm{i}}_{\mathrm{i}} \quad \mathrm{o}_{\mathrm{j}}-\mathrm{ka}_{\mathrm{k}}$-djuuuro
3.INANIM.OBJ 2.SG.ANIM.OBJ-1.SG.ANIM-contar
$E s o_{\mathrm{i}}$ te ${ }_{\mathrm{j}}$ cuento . $_{\mathrm{k}}$

En (2) el pronombre animado catafórico cumple la función sujeto y refiere al nombre al final del enunciado; el indice de persona objeto se prefija a un verbo divalente.

(2) ก̃hã ${ }_{i} \quad \mathbf{x o}_{\mathbf{j}}$-buko?sáña / $\quad \mathbf{k}_{\mathbf{j}}$-onaa-ko $\mathrm{k}_{\mathrm{i}}$

3.ANIM 1.SG.ANIM.OBJ-criar 1.SG.ANIM-hermano.mayor-CLS:'femenino'

Ella $_{\mathrm{i}} \boldsymbol{m e}_{\mathrm{j}}$ crió, mi $\mathbf{j}_{\mathrm{j}}$ hermana mayor . $^{.}$

Los índices pronominales establecen con los nombres a los cuales se prefijan dos tipos de relación. Ésta puede ser necesaria u obligatoria, como en el caso del índice pronominal de primera persona prefijado al nombre 'hermana mayor' en (2), pues éste nombre requiere necesariamente la presencia de otro nombre, que en este caso aparece como una forma pronominal, esta relación puede ser entonces analizada como actancial (ver Fagua, 2013: 155-158 y Queixalós, 2005). El otro tipo de relación entre los nombres es facultativa, como en (3), en este caso el nombre 'trabajo' no requiere obligatoriamente la presencia del prefijo pronominal. A estos dos tipos de relación se refiere también a través de las oposiciones entre nombres alienables vs inalienables o absolutos $v s$ relativos.

\footnotetext{
${ }^{3}$ Los ejemplos presentados han sido tomados del corpus consignado en Seifart, F. et al. (2009) y de enunciados solicitados.
} 


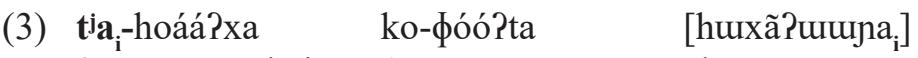 \\ 3.INANIM-trabajo 1.SG.ANIM-querer chacra \\ Me gusta $\boldsymbol{s} \boldsymbol{u}_{\mathrm{i}}$ trabajo [de la chacra $]$.}

Las posposiciones pueden igualmente ser prefijadas con índices personales que remiten a su complemento, como en (4).

\section{(4) baa? ku-mumno aahi \\ acá 1.SG.ANIM-atrás [2.SG.ANIM-]venir}

¡Ven acá atrás de $\boldsymbol{m i ́}$ (lit. mi atrás)!

El género se manifiesta pues como formas pronominales que desempeñan una función anafórica o catafórica. Además, algunos sufijos clasificatorios pueden dar indicios sobre el género semántico de los nombres así marcados, aunque esta relación no es unívoca (ver cuadro 4). En fin, puesto que el género opera una división bipartita del léxico nominal hablamos de una categoría nominal clasificatoria.

\section{Nombres individuales vs genéricos y densos: categoría de número singular}

El tipo de estrategia requerida para referir a entidades discretas divide el léxico nominal de un lado, en nombres individuales y de otro, en nombres genéricos y nombres densos (Culioli, 1985:103; de Vogué, 1989:1-23). Los primeros en su forma de base refieren directamente tanto a la clase o al concepto general como a unidades o individuos (ver 'perro/jaguar" en la figura infra). En tanto que los otros dos en su forma de base refieren a conceptos genéricos o a toda una clase de individuos ( $c f$. no contables) y a fin de referir a entidades discretas deben recurrir a sufijos especializados en la función discretizadora (ver -t to y - $\beta a r a$, en la figura 1), por tal razón son designados como sufijos discretizadores.

Figura 1: Clasificación de nombres según la categoría de número singular

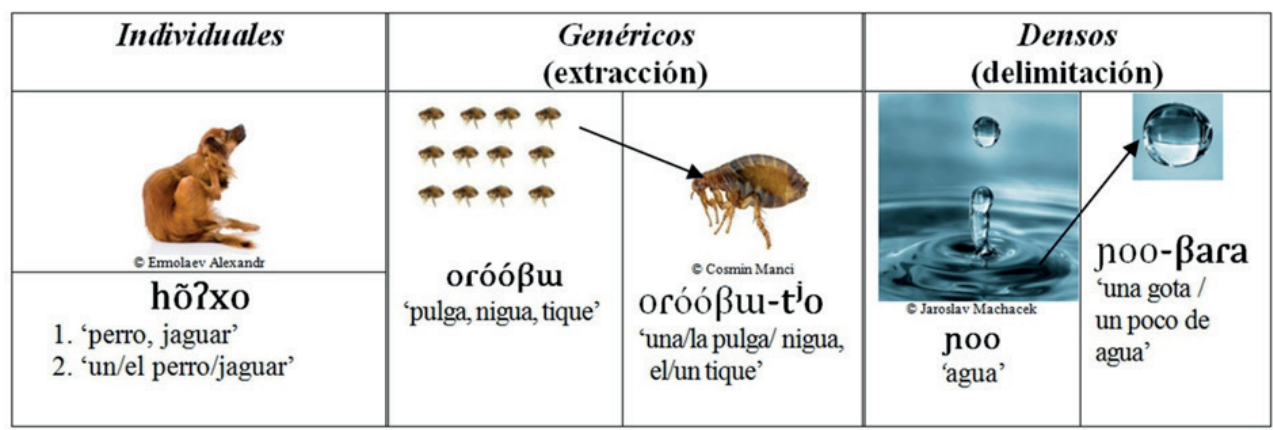


La distinción entre nombres génericos y densos se justifica por el tipo de operación de discretización (Queixalós, 1998:47, 51): extracción en el caso de los nombres genéricos que refieren a toda una clase compuesta por unidades $o$ individuos no especificados en cuanto a la cantidad, es decir no cuantificados, aunque son cuantificables. En el caso de los nombres densos ( $c f$. de masa), la sufijación de las marcas discretizadoras denota la delimitación de una porción que -contrariamente a las unidades resultantes de la operación de extracciónno tienen existencia previa a esa operación de delimitación (ver Figura 1).

El ocaina posee un amplio inventario de sufijos discretizadores, que además son portadores de semantismos específicos. Por interés metodológico, esas cuestiones serán abordadas en la sección 3 .

El cuadro inferior expone un correlato morfosintáctico de la tripartición de los nombres según la categoría de número singular: el sufijo de número dual. En efecto, los nombres individuales reciben directamente la marca de dual; en tanto que los nombres genéricos y densos, previamente a la sufijación de la marca de dual, deben ser discretizados (mediante los sufijos que denotan el número singular).

Cuadro 2: Composición morfológica de los nombres en número dual: correlato morfosintáctico de la tripartición nominal: individual $v s$ genérico y denso

\begin{tabular}{|l|l|l|}
\hline \multicolumn{2}{|c|}{ NOMBRES } \\
\hline \multicolumn{1}{|c|}{ Individuales } & \multicolumn{1}{|c|}{ Genéricos } & \multicolumn{1}{c|}{ Densos } \\
\hline $\begin{array}{l}\text { hõ?xo-?ta } \\
\text { perro/jaguar-DUAL } \\
\text { 'un par de perros/jaguares', }\end{array}$ & $\begin{array}{l}\text { orooßu-tio-Pta } \\
\text { pulga/nigua-CLS-DUAL } \\
\text { 'un par de pulgas, niguas' }\end{array}$ & $\begin{array}{l}\text { jo-ßaráá-Pta } \\
\text { agua-CLS-DUAL } \\
\text { 'dos gotas de agua' }\end{array}$ \\
\hline
\end{tabular}

\section{Los sufijos clasificatorios de discretización}

Como se acaba de exponer, los nombres genéricos y densos en su forma de base no pueden referir directamente a entidades unitarias y por ello recurren a los sufijos discretizadores. Justificaremos ahora nuestro análisis de estos sufijos como clasificadores de la función discretizadora y el rechazo a su análisis como sufijos de clasificación nominal ( $c f$. François, 1999:165-175). En primer lugar, puesto que estos sufijos no determinan la totalidad del léxico nominal, no pueden ser considerados como dispositivos de clasificación de los nombres. Esa afirmación es, en segundo lugar, reforzada por la posibilidad que tienen los nombres genéricos y densos de acoger alternativamente más de una de las marcas que conforman ese paradigma como lo muestran los ejemplos (6 a 8). 
(5) koßáá

uvilla

uvilla (árbol, fruto) ${ }^{4}$

(6) koßaa-ja

uvilla-CLS: 'árbol'

árbol de uvilla.

(7) koßaa-tsouvilla-CLS:'racimo'

un racimo de uvilla.

(8) koßaa-xo

uvilla-CLs: 'oblongo'

un fruto de uvilla.

Parafraseando lo dicho anteriormente, la categoría de número singular marcada a través de los sufijos ilustrados en los ejemplos anteriores solo concierne a una subclase de nombres; contrariamente a lo que ocurre con la categoría de género a la cual se atribuye obligatoriamente todo nombre de la lengua. En vista de que los sufijos discretizadores no determinan la totalidad del léxico nominal, no se puede afirmar que clasifican los nombres.

Lo que François (1999) denomina “efecto clasificatorio”, nos permite ahora comprender el ámbito de la "clasificación” de estos sufijos, que está circunscrito no al léxico nominal en general sino más bien a la manera como las subclases de nombres genéricos y densos pueden ser cuantificadas en unidades, es decir discretizadas. Se justifica así el hablar no de sufijos que clasifican nombres sino de sufijos clasificadores de la operación de discretización que, para abreviar, denominamos sufijos clasificatorios de discretización.

Así, el nombre genérico que denota la clase o concepto general "pez" puede ser cuantificado como individuos adultos o de talla promedio (9), como individuos jóvenes o pequeños (10), o bien como una agrupación de individuos ensartados (11) o incluso como unidades cilíndricas (12). La selección de una $u$ otra marca no es pues obligatoria sino que responde a compatibilidades semánticas y a un referente particular al cual apunta el hablante. Este mismo tipo de explicación es válido para los ejemplos (5 a 8).

(9) ĩhãã-m̦a

pescado-CLS: 'masculino'

un pescado

${ }^{4}$ Pourouma cecropiifolia. 
(10) îhã̃ắ-ßora

pescado-CLS: 'diminutivo.animado'

un pescadito

(11) ĩhãã-tsoro

pescado-CLS: 'sarta, manojo.pequeño'

una sartita de pescado

(12) ĩhãã-kona

pescado-CLS: 'cilíndrico'

una lata de pescado ( $c f$. pescado enlatado)

Podemos ahora introducir otra dimensión que estos sufijos cubren: mediante ellos no solo es posible extraer y delimitar unidades e individuos sino que también contribuyen a especificar los referentes. Entonces es solo después de la sufijación de una marca de ese paradigma que los referentes son identificados y definidos gracias a las propiedades semánticas que estos sufijos vehiculan. En resumen, estos sufijos clasifican las diferentes posibilidades de discretización de conceptos generales; permitiendo así obtener simultáneamente una entidad discreta y un referente más específico.

Una vez entendida la función de este paradigma, pasemos a caracterizarlo en términos de inventario y semantismos. En el cuadro 3 (abajo), se aprecia el listado de las más de 80 marcas discretizadoras identificadas hasta ahora. El inventario está ordenado en función de rasgos semánticos, principalmente propiedades físicas: forma, talla, consistencia, disposición, materia, lo cual pone en evidencia la calificación "clasificatorio" (ver Anexo final). Los sufijos resaltados en gris conjugan más de tres propiedades: así los sufijos -?o, -?(o)ko, $-?(o) \phi e$, de la segunda columna, conjugan las propiedades 'inanimado, tubular o filiforme, grosor, con un tamaños dado' (13 a 15).

(13) ajóóxa-?o

víbora $s p$.-CLs: 'tubular.grosor.medio'

una víbora $s p$.

(14) a?xaаjo-?ko

sachapapa-CLS: 'tubular.angosto'

una liana de sachapapa ${ }^{5}$.

(15) охо-ха-?оофе

comer-NOMZ-CLs: 'filamento'

un espagueti (lit. un filamento de comida).

${ }^{5}$ Discorea sp. 
Finalmente, cabría señalar que el ocaina hace parte de las lenguas que tienen un inventario abundante de marcas clasificatorias de discretización; sin embargo, la cuestión cuantitativa como pudimos comprobarlo en la primera parte, en donde con un puñado de marcas dimos cuenta del fenómeno, no hace un aporte cualitativo respecto a la función que dichas marcas desempeñan. La cantidad de marcas aporta en cambio indicios sobre restricciones, compatibilidades y distinciones semánticas pertinentes en tal o cual lengua. El cuadro 3 ilustra el inventario de estas marcas y su clasificación según diferentes rasgos semánticos. Los sufijos destacados allí con sombreado gris combinan más de tres rasgos semánticos (ver Anexo final para precisar el significado de las marcas).

\section{Interacción entre los sistemas de género y número}

Por último, es de resaltar la correlación simétrica que existe entre los sistemas de clasificación de los nombres según las categorías de género y número. En efecto, los nombres individuales son siempre animados y los nombres densos inanimados; mientras los nombres genéricos pueden ser animados o inanimados, dependiendo del referente en cuestión. Eso es lo que la siguiente figura pone en evidencia.

Figura 2. Clasificación de los nombres según género y número

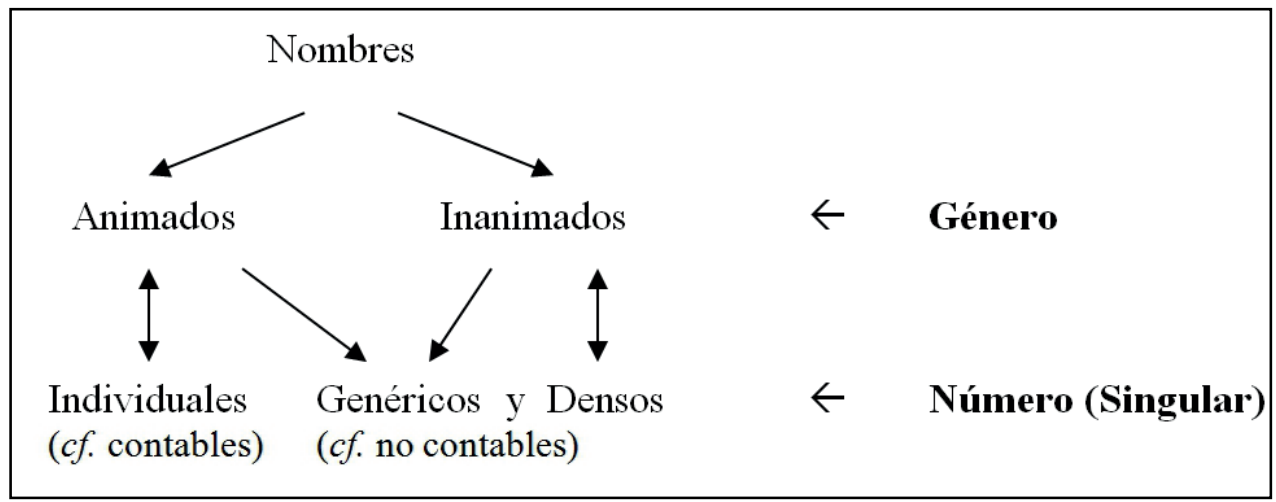

En el siguiente cuadro se ilustra la interacción entre las categorías de género: animado vs inanimado; y número singular: nombres individuales $\mathrm{y}$ nombres genéricos y densos, discretizados gracias a marcas especializadas en la función discretizadora (delimitación y extracción). Cabe recordar que las marcas clasificatorias de discretización presentan restricciones combinatorias en función de las propiedades semánticas de los nombres a los cuales se sufijan: los nombres densos, por ejemplo, son especificados únicamente por los sufijos clasificatorios general o aquellos que remiten a líquidos. 


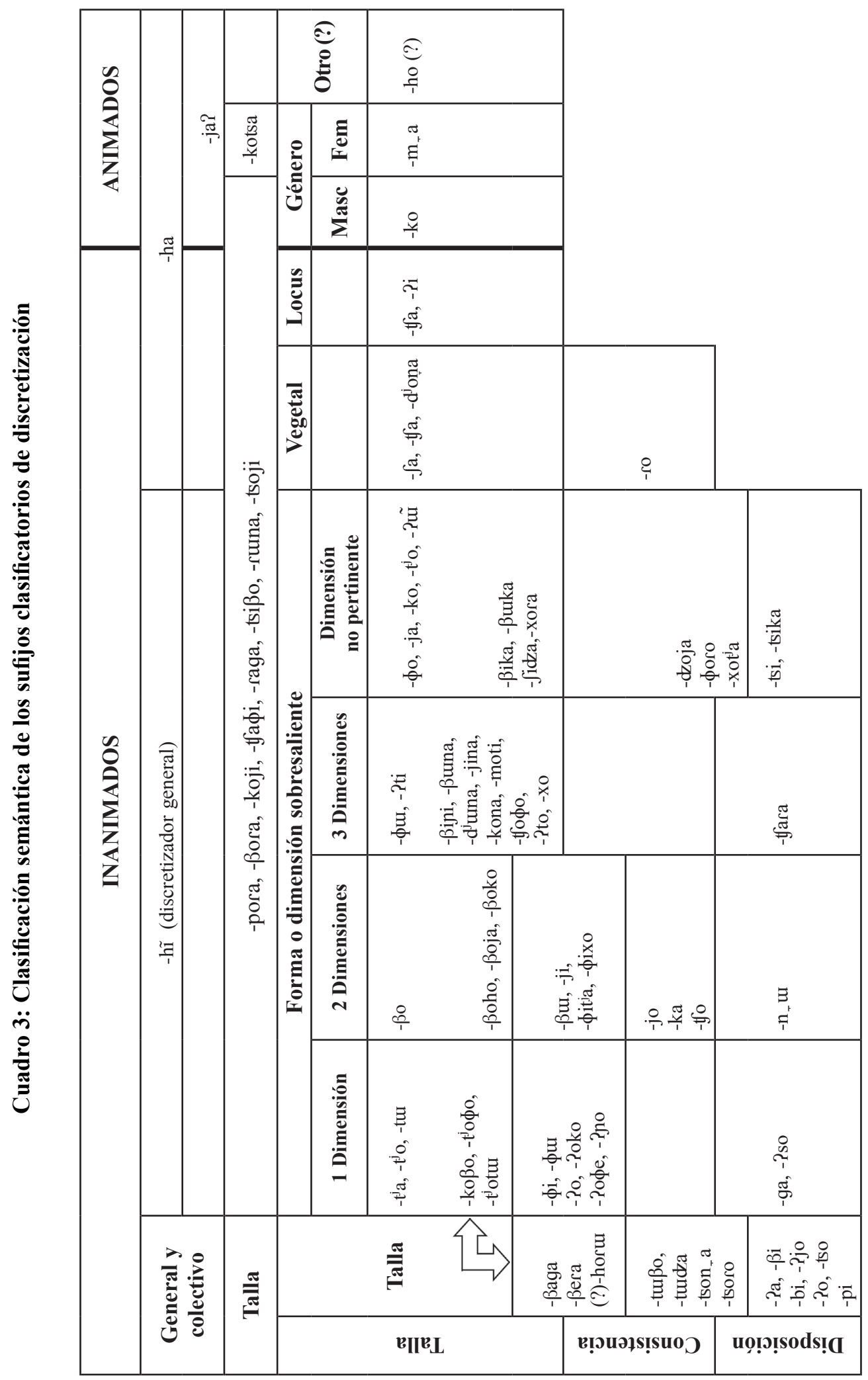


Cuadro 4. Interacción entre las categorías de género y número singular

\begin{tabular}{|c|c|c|}
\hline Género & Animados & Inanimados \\
\hline \multirow{3}{*}{ 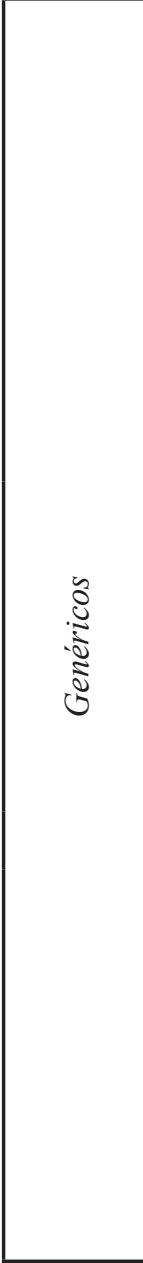 } & $\begin{array}{l}\text { orooßu pulga, chinche, pescadito } s p \text {. } \\
\text { orooßu-tio una/la pulga, un/el chinche, } \\
\text { pescadito } s p \text {. } \\
\{\text { N-CLS: 'alargado'\} } \\
\text { goraa hormiga comestible } \\
\text { goraa-ma una hormiga } \\
\{\text { N-CLS: 'masculino'\} }\end{array}$ & $\begin{array}{l}\text { a?tĩĩ diente(s) } \\
\text { a?ti-Ptio un diente } \\
\text { \{N-CLS: 'alargado'\} } \\
\text { obãã ungurahui (Oenocarpus bataua) } \\
\text { obãã-Pto un fruto de ungurahui } \\
\{\text { N-CLS:‘oblongo'\} } \\
\text { nii chambira (Astrocarium chambira) } \\
\text { ní-Poфe una/la fibra de chambira } \\
\{\mathrm{N}-\mathrm{CLS}: \text { 'filamento'\} }\end{array}$ \\
\hline & $\begin{array}{l}\text { õhõõ-ma un joven, muchacho } \\
\text { õhõõ-ko una joven, muchacha } \\
\text { \{N-CLS: 'femenino'\} } \\
\text { manii-?xo una tortuga } \\
\text { \{N-CLS: 'contorno redondeado, pequeño'\} } \\
\text { hojaa-ko una garrapata }\end{array}$ & $\begin{array}{l}\text { gotúú-?xo una piedra, guijarro } \\
\text { hojáá-ßoko un moneda / dinero } \\
\text { \{N-CLS: 'aplanado'\} }\end{array}$ \\
\hline & 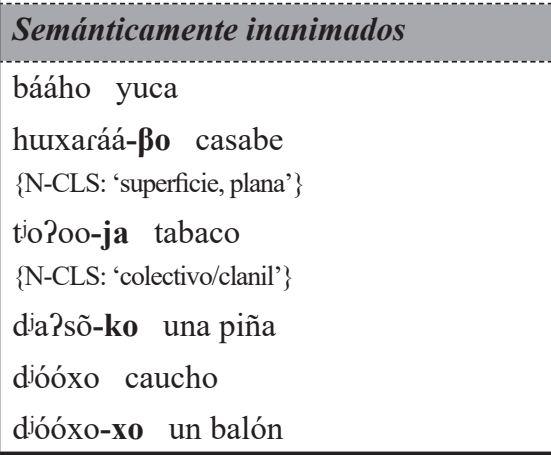 & $\begin{array}{l}\text { hííbi coca } \\
\text { hííbi-ro una planta de coca } \\
\text { \{N-CLS: 'alargado y flexible'\} }\end{array}$ \\
\hline $\begin{array}{l}\tilde{0} \\
\vdots \\
\vdots \\
0\end{array}$ & & $\begin{array}{l}\text { noo agua } \\
\text { noo-Bara una gota de agua } \\
\text { agua-CLS: 'líquido, pequeña porción' } \\
\text { noo-hũ un poco de agua, aguas } \\
\text { \{N-CLS: 'general'\} } \\
\text { nomaa-hĩ la canción, canciones } \\
\text { \{canto/ar-CLS: 'general'\} } \\
\text { tioaa-hĩ la enfermedad, enfermedades } \\
\text { \{enfermar-CLS: 'general'\} }\end{array}$ \\
\hline 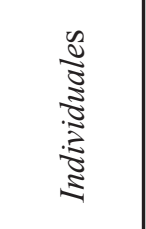 & $\begin{array}{l}\text { oo?u (un / el) hombre } \\
\text { haró?tiba (un / el) pécari } \\
\text { aam̃i (un / el) mono nocturno (chosna) } \\
\text { nõõũ (un / el) murciélago } \\
\text { koono (un / el) perdiz }\end{array}$ & \\
\hline
\end{tabular}


El segmento del cuadro sombreado en gris corresponde a nombres semánticamente inanimados, pero concebidos en esta lengua como animados (16). Se trata de entidades del mundo vegetal que han sido domesticadas y sus productos derivados. Esta explicación permite dar cuenta del tratamiento diferencial, en cuanto a la atribución valores del género de dos de los elementos fundadores de la Gente de centro, complejo cultural al cual pertenecen los ocainas: la coca (inanimado) y tabaco (animado). Lo anterior aporta por lo demás pruebas lingüísticas para confirmar la hipótesis de la adopción tardía de la coca por parte de estos pueblos, permitiendo asimismo corroborar la cuestión de la frontera fluida entre Andes (de donde provendría la coca) y la Amazonia (Lathrap, 1982:92-94).
(16) [bóótfo $\left.o_{j}\right]$ ĩ̃hã na-háá -noxo?ta?úna
cahuana
3.SG.ANIM.OBJ
3.SG.ANIM.OBJ-3.PL.ANIM-beber
A él ${ }_{i}$ se $\mathbf{l a}_{\mathrm{j}}$ hacían $_{\mathrm{k}}$ beber [la cahuana $\mathbf{j}_{\mathrm{j}}$ (bebida de almidon de yuca)].

En síntesis, en ocaina la categoría de género distribuye el léxico nominal en dos clases, según su atribución obligatoria a uno de los dos valores: +/- animado; esta atribución se manifiesta como marcas de concordancia pronominales que en función anafórica o catafórica retoman los nombres que controlan tal concordancia. Se trata pues de un dispositivo clasificatorio del léxico nominal. Contrariamente, el recurso a los sufijos discretizadores no es obligatorio, pues solo concierne a una subclase de nombres. A esto se suma el hecho de que un nombre puede ser sufijado con más de una de las marcas discretizadoras, según el referente en cuestión. Por lo anterior tales sufijos no pueden ser considerados como un dispositivo clasificatorio de los nombres. El paradigma de sufijos discretizadores permite extraer o delimitar unidades o individuos, específicados según las propiedades físicas o funcionales que los sufijos indican. En otras palabras, gracias a la sufijación de estas marcas no se obtienen indicaciones sobre propiedades físicas o funcionales de los nombres, sino sobre la manera en la que tales nombres pueden ser cuantificados como unidades. Las unidades discretas resultantes constituyen por lo demás referentes mejor especificados e identificables, lo cual contribuye a seguir la referencia en el discurso ${ }^{6}$.

Para concluir podemos dejar abierta la vía de contemplar lo expuesto anteriormente como una alternativa posible de análisis de los fenómenos de clasificación nominal, en particular, pero tal vez no exclusivamente, de las lenguas de la misma área lingüística. Así por ejemplo, la división de marcadores

\footnotetext{
${ }^{6}$ La presente exposición deja de lado aspectos que han sido abordados en otro artículo que trata desde una perspectiva más amplia la cuestión de la clasificación nominal: "Sintagma y clasificación nominales en ocaina (witoto): clases de nombres, género y sufijos clasificatorios de discretización" (Fagua, en prensa).
} 
de clase propuesta por Seifart (2005:77), a saber: marcas de clasificación generales y marcas específicas, podría groso modo coincidir respectivamente con las distinciones de género gramatical y de sufijos clasificatorios que proponemos aquí.

\section{Abreviaturas y convenciones}

$\begin{array}{ll}- & \text { valores concomitantes } \\ - & \text { Sufijo } \\ \text { ANIM } & \text { Animado } \\ \text { CLS } & \text { sufijo clasificatorio de discretización } \\ \text { INANIM } & \text { inanimado } \\ \text { lit. } & \text { literalmente } \\ \text { NOMZ } & \text { nominalizador } \\ \text { OBJ } & \text { objeto } \\ \text { POSP } & \text { posposición } \\ \text { PL } & \text { plural } \\ \text { SG } & \text { singular }\end{array}$

\section{Anexo}

Semantismo de los sufijos clasificatorios de discretización

\begin{tabular}{|l|l|}
\hline \multicolumn{2}{|c|}{ NOMBRES ANIMADOS E INANIMADOS } \\
\hline Sufijo general de número o clasificación \\
\hline -hĩ & (número) general, inanimado \\
\hline Quanta & \\
\hline -ha & colectivo (in)animados \\
\hline Talla (por derivación apreciación) & \\
\hline -ßßora (cf. -runa) & $\begin{array}{l}\text { pedazo, porción pequeña (diminutivo, } \\
\text { hipocorístico) }\end{array}$ \\
\hline -tsißo & pequeño (diminutivo, hipocorístico) \\
\hline -runa (cf. diuna, moti, -xo, -so, -to) & oblongo (blando) (aumentativo, peyorativo) \\
\hline -tsoji & pequeño (diminutivo, hipocorístico) \\
\hline
\end{tabular}

\begin{tabular}{|l|l|}
\hline \multicolumn{2}{|c|}{ NOMBRES INANIMADOS } \\
\hline Quanta & \\
\hline ja? & colectivo, humano \\
\hline
\end{tabular}




\begin{tabular}{|l|l|}
\hline Talla (por derivación apreciación) & \\
\hline -kotsa (cf. -ko, - $\beta 0$ a) & femenino, anciano \\
\hline Género semántico & \\
\hline -ko (cf. -kotsa, -ma) & femenino \\
\hline -ma (cf. -ko, -kotsa) & masculino \\
\hline Otros (?) & \\
\hline -ho & animal (¿sílaba epentética?) \\
\hline
\end{tabular}

\begin{tabular}{|c|c|}
\hline \multicolumn{2}{|c|}{ NOMBRES INANIMADOS } \\
\hline \multicolumn{2}{|c|}{ Talla (apreciación, por derivación) / Cantidad } \\
\hline -pora (cf. -ßora) & pequeño (?) \\
\hline -koji & (?), diminutivo, hipocorístico \\
\hline$-\mathbf{t} \mathbf{a} \phi \mathbf{i}$ & pedazo, parte \\
\hline -raga & aumentativo, peyorativo \\
\hline \multicolumn{2}{|c|}{ Talla (apreciación, por derivación) y consistencia } \\
\hline 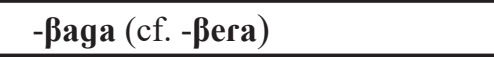 & líquido denso/ aumentativo (peyorativo) \\
\hline 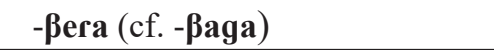 & líquido, diminutivo, hipocorístico \\
\hline (?) -horu (cf. hooru 'tamizar') & tamizado, granulado \\
\hline$-\mathbf{a} \mathbf{a}(\mathrm{cf} .-\boldsymbol{\beta} \mathbf{i},-\mathrm{bi})$ & en filas, rangos \\
\hline$-\beta \mathbf{i}(\mathrm{cf} .-\mathbf{- 2},-\mathrm{bi})$ & en filas, rangos (estrechos) \\
\hline$-b \mathbf{i}(\mathrm{cf} .-\mathbf{- 2},-\boldsymbol{\beta i})$ & par de objetos agudos (paralelos) \\
\hline -?jo (cf. -tuudza) & bastoncillos/láminas entretejidas \\
\hline-20 & racimo (en el árbol), ensartado \\
\hline -tso (cf. -Po) & ramo, ramillete \\
\hline$-\mathbf{p i}$ & rizoma \\
\hline$-\operatorname{tw\beta } \beta$ & haz de filamentos/fibras/hilos \\
\hline -tudka (cf. - -2jo) & bastoncillos, entretejidos \\
\hline -tsoña (cf. -tso, -tsoro) & vellos, pelusa \\
\hline -tsoro (cf. -tso, -tsona) & haz/manojo, pequeño \\
\hline \multicolumn{2}{|c|}{ Forma sobresaliente de una dimensión } \\
\hline$-\mathbf{t}^{\mathrm{j}} \mathbf{a}\left(\mathrm{cf} .-\mathrm{t}_{\mathrm{j}}^{\mathrm{j}},-\mathrm{-t}_{\mathrm{j}}^{\mathrm{j}} \phi \mathbf{0},-\right.$ kona, - -ro) & tubular, agudo \\
\hline 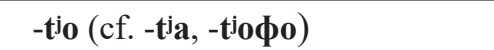 & tubular, aguda, (in)animado \\
\hline -twu (cf. -tiotu) & extremidad \\
\hline$-k o \beta o\left(\right.$ cf. $\left.-\mathbf{t}^{\mathrm{j}} \mathbf{a},-\mathbf{t}^{\mathrm{j}} \mathbf{0} \phi \mathbf{0}\right)$ & tubular \\
\hline$\left.-\mathbf{t}^{\mathrm{j}} \mathbf{0} \phi \mathbf{( c f .}-\mathbf{t}^{\mathrm{j}} \mathbf{\mathrm { j }},-\mathrm{t}^{\mathrm{j}} \mathbf{a}\right)$ & cónico, pequeño \\
\hline
\end{tabular}




\begin{tabular}{|c|c|}
\hline 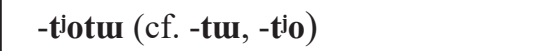 & gran extremidad \\
\hline$-g a$ & soporte \\
\hline- Pso & haz de objetos picantes \\
\hline 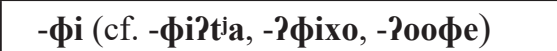 & filamento(s) \\
\hline$-\phi ш$ & prominente, flexible \\
\hline-20 & tubular, estrecha, flexible (liana) \\
\hline- Poko (cf. - $\phi \mathbf{i},-$-Po) & tubular, talla media (liana) \\
\hline -20oфe (cf. -фi, -Po) & filamento (suspendido) \\
\hline- -2no & alargado, estrecho \\
\hline \multicolumn{2}{|c|}{ Forma sobresaliente de dos dimensiones } \\
\hline$-\beta o($ cf. $-\boldsymbol{\beta} u,-\beta o j a)$ & superficie, plana \\
\hline$-\beta o h o($ cf. $-\beta o j a,-\beta o,-\beta u)$ & $\begin{array}{l}\text { plano, relativamente amplio (triangulare, } \\
\text { trapezoidal) }\end{array}$ \\
\hline 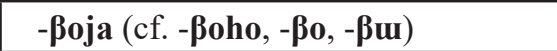 & plano, estrecho (triangular, trapezoidal) \\
\hline 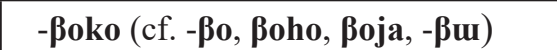 & plano, estrecho, redondeado \\
\hline$-\mathbf{j o}$ & capa, forro \\
\hline$-\mathbf{k a}$ & envoltura \\
\hline$-1 \mathrm{fo}$ & redondeado, irregular \\
\hline$-n_{i} \mathbf{u}$ & trenzado \\
\hline 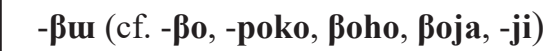 & plano, laminar (rectangular, oval, trapezoidal) \\
\hline -ji (cf. - $\beta \mathbf{u})$ & laminar (alas, hojas (?)) \\
\hline 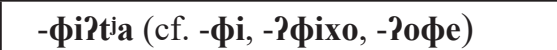 & tejido, fino \\
\hline$-? \phi i x o$ (cf. $-\phi i ? \mathrm{t} j a)$ & tejido (hipocorístico) \\
\hline \multicolumn{2}{|c|}{ Forma sobresaliente de tres dimensiones } \\
\hline$-\phi u$ & contenedor \\
\hline -Pti (cf. -jina, -moti) & esférico \\
\hline$-\beta \mathbf{i n i}$ & oval, romo, pequeño \\
\hline -ßßuna (cf. -djuna, -runa, -kona) & cilíndrico, grande \\
\hline -djuna (cf. -ßuna, -runa) & esférico/oval, talle media \\
\hline -jina (cf. -Pti, -moti, -djuuna) & esférico, grande \\
\hline -kona (cf. - $\mathbf{t}^{\mathrm{t}} \mathbf{a},-$-ro) & tubular, estrecho \\
\hline -moti (cf. -jina, -ti, -xo, -so, -to) & esférico/oblongo, pequeño \\
\hline -tfoфo (cf. -funa antonyme) & cono, (recipiente) cónico \\
\hline- Pto (cf. -xo) & oblongo, pequeño \\
\hline$-\mathbf{x o}$ (cf. -?to) & contorno redondeado, pequeño \\
\hline -tfara & en espiral, caracol \\
\hline
\end{tabular}




\begin{tabular}{|c|c|}
\hline \multicolumn{2}{|c|}{ Forma sobresaliente (dimensión no pertinente) } \\
\hline -фо (cf. фоо 'maison') & cóncavo \\
\hline$-\mathbf{j a}$ & contenedor (grande) (?)' \\
\hline -ko (cf. -xo, -Pto) & contorno, redondeado, pequeño (?) \\
\hline 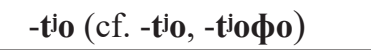 & cóncavo \\
\hline$-2 \tilde{u}$ & prominencia \\
\hline 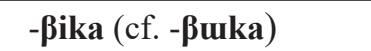 & prominente, curvo, exterior, cóncavo \\
\hline - $\beta$ uka (cf. $\beta$ ika) & prominente, curvo, exterior \\
\hline$-\int \mathbf{i d z a}$ & pequeño/hipocorístico \\
\hline -xora (cf. -xo, -xot'ja) & contenedor cilíndrico \\
\hline -tsi (cf. -tsika) & hendido, con hendiduras \\
\hline -tsika (cf. -tsi) & estructura con hendiduras/orificios \\
\hline -dzoja & haz, prominente, curvo, exterior, tendido \\
\hline -фоro & haz, prominente, curvo, exterior, flexible \\
\hline$-\operatorname{xotj}^{\mathrm{j}}(\mathrm{cf} .-\mathrm{xo},-$-xora $)$ & contenedor redondeado, flexible \\
\hline \multicolumn{2}{|l|}{ Locus } \\
\hline$-\mathbf{t g a}$ & lugar (?) \\
\hline$-\mathbf{i}$ & medio de transporte (?) \\
\hline \multicolumn{2}{|l|}{ Vegetal } \\
\hline$-\int \mathbf{a}$ & $(?)$ \\
\hline -djoña & $(?)$ \\
\hline$-\mathrm{tga}$ & $(?)$ \\
\hline \multicolumn{2}{|l|}{ Vegetal y consistencia } \\
\hline -ro (cf. -kona, - - $\left.\mathrm{t}^{\mathrm{j} a}\right)$ & arbusto, tubular, blando \\
\hline
\end{tabular}

\section{Referencias}

Aikhenvald, Alexandra Y. 2011. Classifiers. In: Oxford Bibliography Online. Oxford University Press, New York, NY, USA: 1-40.

Corbett, Greville. 2007. Gender and noun classes. Language typology and syntactic description. New York. Cambridge University Press. Vol 3: 241-279.

Culioli, Antoine. 1985. Notes du séminaire de DEA 1983-84. Université Paris 7.

De Vogué, Sarah. 1989. Discret, dense, compact: les enjeux énonciatifs d'une typologie lexicale. La notion de prédicat. Paris, Université Paris 7: 1-23.

Fagua, Doris. 2013. Aspects morphosyntaxiques de l'ocaina. Autour des classes lexicales. Tesis doctoral, Universidad de París 7, Denis Diderot. 
Fagua, Doris. [en prensa] "Sintagma y clasificación nominales en ocaina (witoto): clases de nombres, género y sufijos clasificatorios de discretización".

François, Alexandre. 1999. "L’illusion des classificateurs". Faits de langues $\mathrm{N}^{\circ} 14$ : $165-175$.

Grinevald, Colette. 2004. "Noun Classes in African and Amazonian languages : towards a comparison". Linguistic typology $N^{\circ} 8, \mathrm{~N}^{\circ} 2: 243-285$.

. 2002. "Making sense of nominal classification systems. Noun classifiers and grammaticalization variable". Ilse Wischer \& Gabriele Diewald (Eds.). New reflections on Grammaticalization. Series: Typological studies in language, Vol. 49. Amsterdam/Philadelphia, John Benjamins B. V.: 259-275.

Lathrap, Donald. 1982. "La antigüedad e importancia de las relaciones de intercambio a larga distancia en los trópicos húmedos de Sudamérica precolombina". En: Amazonia peruana, 4 (7): 79-97.

Petersen de Piñeros, Gabriele. 2007. Nominal classification in Uitoto. International Journal of American Linguistics 73(4): 389-409.

Queixalós, Francesc. 2005. "Posse em Katukina e valência dos nomes". Novos estudos sobre línguas indígenas. Brasilia, Editora UnB: 177-202. . 1998. Nom, verbe et prédicat en sikuani (Colombie). Louvain/Paris, Peeters.

Seifart, Frank, Doris Fagua, Jorge Gasché \& Juan Echeverri. 2009. A multimedia documentation of the languages of the People of the Center. Online publication of transcribed and translated Bora, Ocaina, Nonuya, Resigaro, and Witoto audio and video recordings with linguistic and ethnographic annotations and descriptions. Nijmegen, DoBeS-MPI. http://corpus1.mpi.nl/ds/imdi_ browser/?openpath=MPI77915\%23.

Seifart, Frank. 2005. The structure and use of shaped-based noun classes in Miraña (North West Amazon). Nijmegen: Max Planck Institute for Psycholinguistics : 77-179.

Seifart, Frank \& Doris L. Payne. 2007. Nominal Classification in the North West Amazon: Issues in Areal Diffusion and Typological Characterization. International Journal of American Linguistics 73 (4): 381-387.

Data recebimento: $17 / 02 / 2014$.

Data aceite: $18 / 04 / 2014$. 